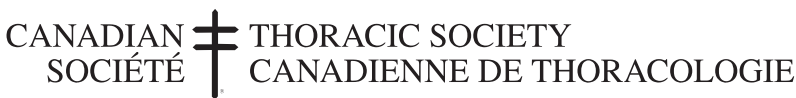

\section{The next steps}

Tam humbled to assume the presidency of the Canadian Thoracic Society (CTS). As I contemplate the achievements of my predecessors, I recognize that they have built a strong and vibrant organization, which has become a world leader in the development of clinical practice guidelines (CPGs), is in the midst of developing and implementing a national respiratory research agenda and is creating high-calibre continuing professional development programs. I will continue to support the CTS' development in these key areas, but also plan to ensure that we continue to translate our many achievements into improvements in medical practice and measurable impacts on respiratory health outcomes. It is clear to me that to do so, we will need to enhance our involvement in education, knowledge translation (KT) and evaluation.

I have been involved with the CTS for many years and recall earlier studies such as "Asthma control in Canada remains suboptimal: The Reality of Asthma Control (TRAC) study" (1), which showed that, despite the existence of outstanding CPGs, health outcomes were not improving. This message did not fall on deaf ears! The CTS Executive, and the Professional Development, Research and Guidelines committees are well aware of these findings, and while limits in human and financial resources have slowed our pace, we have always been cognizant of these concerns.

I believe that as an organization, we are finally ready to tackle these lingering issues in a more concerted fashion. I believe that we now have the capacity to begin the process of enhancing our education, KT and evaluation strategies to bridge the gap between research and CPGs, and real-life practice and health outcomes.

It is obvious to me that we are already well underway:

- The CTS implemented a new streamlined governance structure that will more effectively support its three pillars: research, guidelines and education.

- As part of the new governance structure, we have a strong Education and Professional Development Committee with a mandate to guide the development of all CTS educational programs, and to evaluate these programs and their outcomes.

- The 2010 Canadian Lung Association Leadership Conference enshrined research as a core priority of the organization; this is very significant because research will play a vital role in any of our efforts to develop evidence-based CPGs and evaluate their implementation and impact.

- We have created a full-time position for a manager of continuing professional development, and have hired Richard McCoy - an experienced professional - who is positioned to support existing and, possibly, new educational programs.

- Our Canadian Respiratory Guidelines Committee (CRGC) recently created the Knowledge Translation Committee and developed a strategic plan that clearly states its intention to have an impact on the lives of Canadians.

- According to this new strategic plan, the CRGC will guide the development of innovative KT tools and provide access to such tools to facilitate the uptake of our CPGs. Furthermore, with the assistance of communication experts, we will translate scientific evidence into market-ready information for our members, the general public and governments. There are also plans to collaborate with provincial lung associations on the development of guidelines for patients.

\section{Les prochaines étapes}

C'est avec grande humilité que j'aborde la présidence la SCT. Les Créalisations de mes prédécesseurs sont considérables. Ils ont érigé une organisation solide et dynamique de renommée internationale dans le domaine de l'élaboration de lignes directrices, qui travaille à la conception et à la mise en œuvre d'un ordre du jour national pour la recherche en santé respiratoire et à la creation de programmes de développement professionnel continu de haut calibre. Je donnerai mon appui à toutes ces activités clés, mais je chercherai également à m'assurer que ces réalisations continuent à se traduire en améliorations concrètes dans les pratiques médicales et les résultats pour la santé. Afin d'y arriver, il nous faudra sans doute renforcer notre implication dans les domaines de l'éducation, de l'application des connaissances et de l'évaluation.

Je suis membre actif de la SCT depuis de nombreuses années et je me souviens d'études, telles que l'étude TRAC (Asthma control in Canada remains suboptimal: The Reality of Asthma Control) qui démontraient que malgré l'existence d'excellentes lignes directrices pour la pratique clinique, les résultats sur la santé ne s'amélioraient guère. Nous ne sommes pas resté sourds à ces conclusions. Les comités exécutif, de développement professionnel, de recherche et de lignes directrices de la SCT y demeurent attentifs et malgré l'impossibilité d'y donner suite immédiatement (en raison de ressources humaines et financières insuffisantes), ils en ont toujours tenu compte.

Je crois que la SCT est enfin prête à se pencher plus délibérément sur ces questions. À mon avis, nous sommes maintenant en mesure d'entamer un processus d'amélioration de nos stratégies en matière d'éducation, d'application des connaissances et d'évaluation afin de combler l'écart entre, d'une part, la recherche et les lignes directrices et d'autre part, les pratiques réelles et les résultats sur la santé.

Je constate que le travail est déjà bien entamé :

- La SCT a mis en œuvre une nouvelle structure de gouvernance rationalisée, plus apte à soutenir les trois piliers de la SCT, soit la recherche, les lignes directrices et l'éducation.

- Cette nouvelle structure de gouvernance a permis la création d'un solide comité d'éducation et de développement professionnel continu, chargé d'orienter l'élaboration de tous les programmes éducatifs de la SCT et d'en évaluer l'efficacité.

- La Conférence sur le leadership de l'Association pulmonaire du Canada a confirmé que la recherche est une que priorité fondamentale de l'association. Cette décision est de grande portée car la recherche jouera un rôle crucial dans l'élaboration de lignes directrices fondées sur les preuves, ainsi que dans l'évaluation de leur efficacité.

- Nous avons créé un poste à plein temps pour un gestionnaire en développement professionnel continu et avons embauché Richard McCoy, un professionnel chevronné, qui donnera un appui à tous nos programmes éducatifs et pourait en élaborer de nouveaux;

- Notre Comité des lignes directrices canadiennes en santé respiratoire (CLDSR) a mis sur pied un comité sur l'application des connaissances et a élaboré un plan stratégique qui énonce clairement son intention d'avoir un impact dans la vie des Canadiens et des Canadiennes.

- Conformément à ce nouveau plan stratégique, le CLDSR favorisera l'élaboration d'outils innovateurs et les rendront accessibles afin d'en faciliter la mise en application. De plus, avec l'aide d'experts en communications, nous élaborerons des produits d'information ciblés pour nos membres, le grand public et les gouvernements. Il y a aussi des plans provisoires d'élaborer des lignes directrices à l'intention 
- The annual guidelines update will continue to be delivered (as an accredited continuing medical education event), and the CRGC will strive to integrate respiratory CPGs in research sessions offered at the Canadian Respiratory Conference and other key meetings.

- The CRGC will identify new funding opportunities to sustain current operations, fund new strategic KT initiatives, and engage key stakeholders from across Canada to enhance the use of guidelines and foster the development of effective educational programs based on our CPGs.

- Another significant goal of the CRGC is to evaluate health and patient outcomes. We will, therefore, develop evaluation activities to measure the effects of our CPGs on health outcomes.

We certainly have a great headstart on bridging the gap between evidence-based research and CPGs, and real-life medical practice and health outcomes! I plan to support all of these efforts and, in particular, I aim to ensure that through these efforts, we facilitate the delivery of tools, resources and programs that support the professional development of our members - leaders in respiratory medicine who work on the front lines of the health care system all across Canada.

I look forward to working with the CTS Committees, their members, and stakeholders from across Canada to strengthen our collective impact on education, KT and evaluation, to ensure that our activities have the desired impact on the practices of physicians and other health professionals, and on health outcomes.

Together, I am sure we can bridge the gap!

Respectfully submitted, George A Fox MD MSc FRCPC FCCP President, Canadian Thoracic Society

\section{REFERENCE}

1. FitzGerald JM, Boulet LP, McIvor RA, Zimmerman S, Chapman KR. Asthma control in Canada remains suboptimal: The Reality of Asthma Control (TRAC) study. Can Respir J 2006;13:253-9. des patients en collaboration avec les associations pulmonaires provinciales.

- La mise à jour annuelle des lignes directrices aura toujours lieu (en tant qu'activité agréée de formation médicale continue) et le CLSDR favorisera l'incorporation des lignes directrices dans les séances sur la recherche du Congrès canadien sur la santé respiratoire et d'autres congrès clés.

- Le CLSDR identifiera de nouvelles sources de financement afin de soutenir les programmes existants et de soutenir de nouvelles initiatives stratégiques, ainsi que de mobiliser les intervenants pour trouver des moyens de rehausser la mise en application des lignes directrices et de stimuler l'élaboration de programmes éducatifs efficaces fondés sur ces dernières.

- Le CLSDR vise à atteindre un autre but de grande importance : évaluer les résultats sur la santé et sur le patient. Nous élaborerons donc des moyens d'évaluer l'impact de nos lignes directrices sur la santé.

Nous sommes sur la bonne voie dans nos tentatives de combler le fossé entre la recherche et les lignes directrices, et les pratiques réelles et les résultats sur la santé! J'appuierai tous ces efforts et je m'assurerai aussi que, tout en poursuivant ces objectifs, nous favoriserons l'accès à des outils, des ressources et des programmes qui appuient le développement professionnel de nos membres, soit des leaders dans la médecine respiratoire, affectés aux services de santé de première ligne, partout au Canada.

J'ai hâte de travailler avec les comités de la SCT et leurs membres, ainsi qu'avec des intervenants de partout au Canada afin de rehausser notre impact global sur l'éducation, l'application des connaissances et l'évaluation. Nous ferons en sorte que nos activités aient l'impact souhaité sur les pratiques des médecins et d'autres professionnels de la santé, ainsi que sur la santé.

Ensemble, nous arriverons à faire le pont!

Salutations distinguées, George A Fox MD MSc FRCPC FCCP, Président, Societé canadienne de thoracologie

\section{RÉFÉRENCE}

1. FitzGerald JM, Boulet LP, McIvor RA, Zimmerman S, Chapman KR. Asthma control in Canada remains suboptimal: The Reality of Asthma Control (TRAC) study. Can Respir J 2006;13:253-9. 


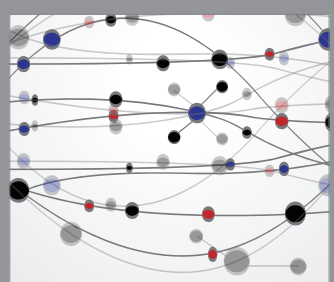

The Scientific World Journal
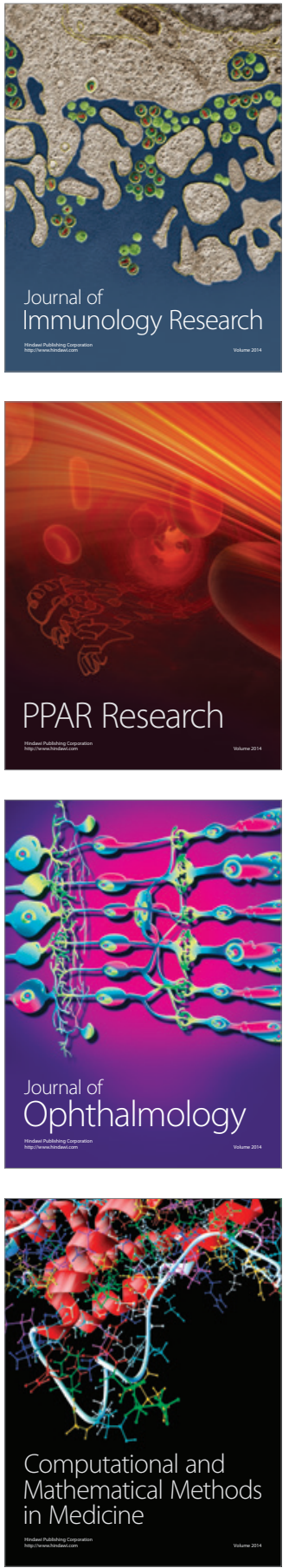

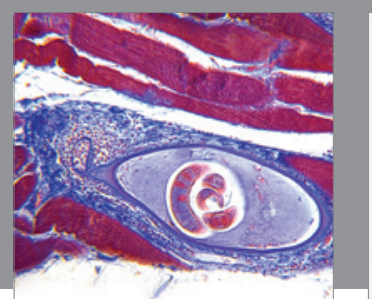

Gastroenterology Research and Practice

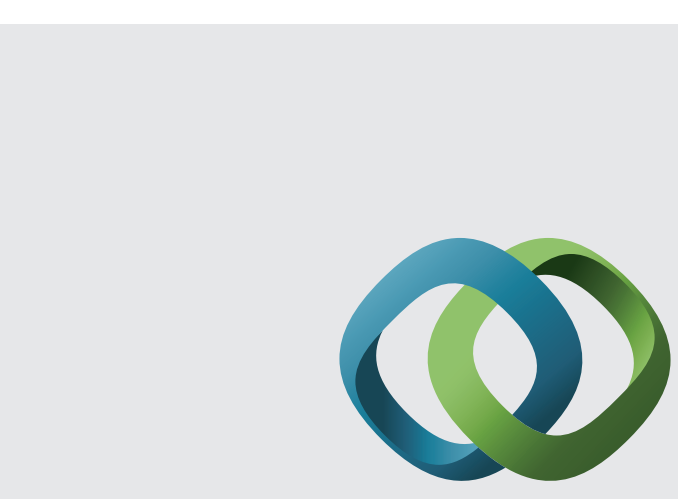

\section{Hindawi}

Submit your manuscripts at

http://www.hindawi.com
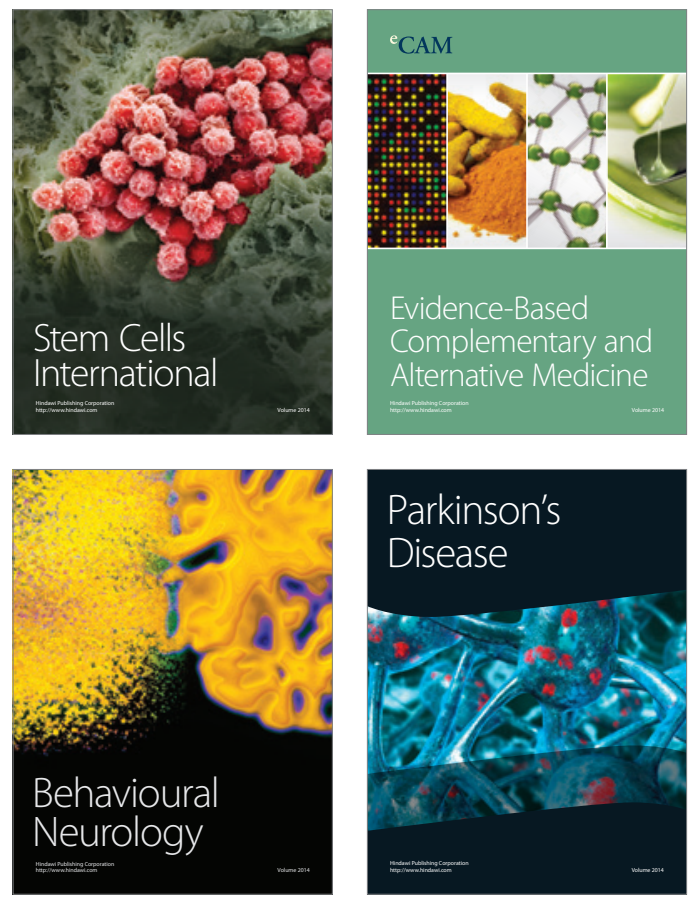
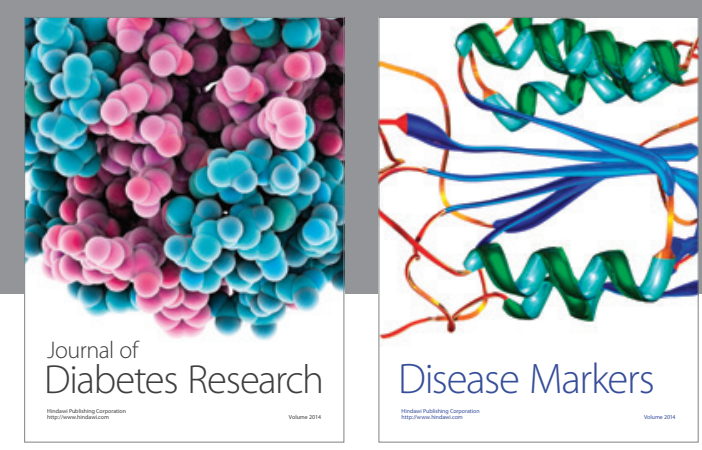

Disease Markers
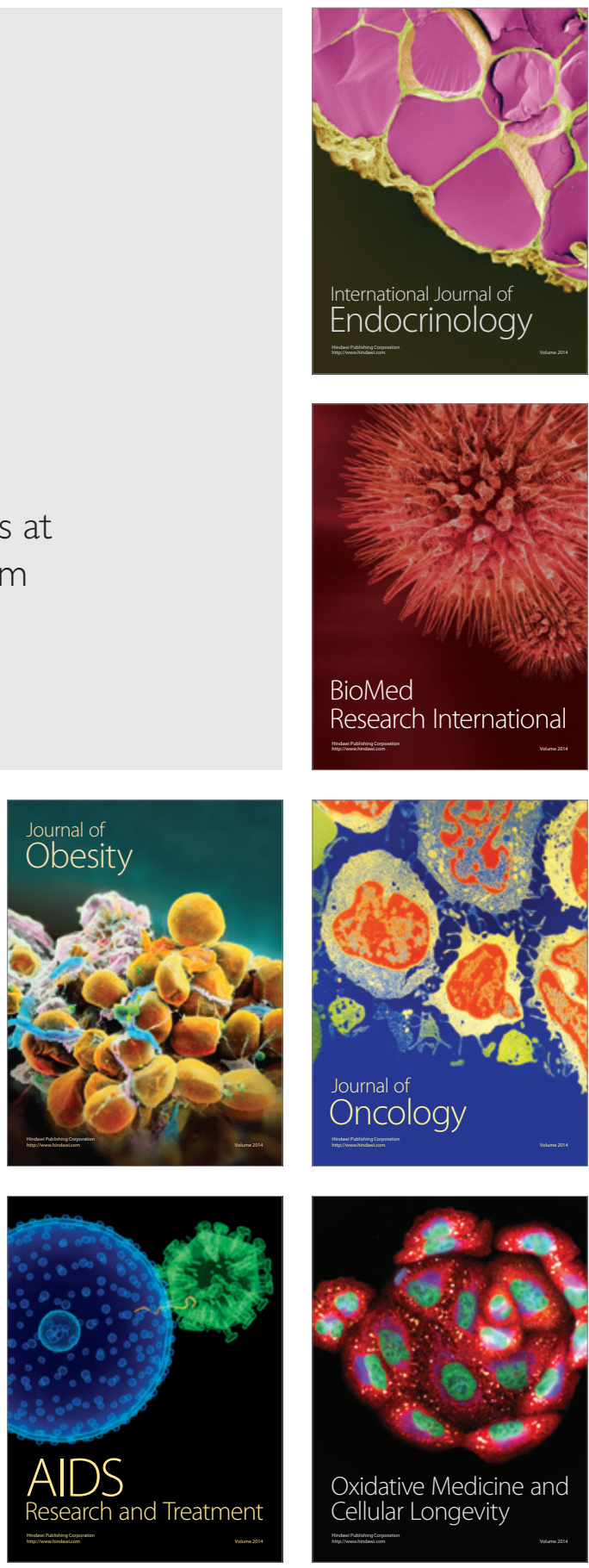